\title{
French proper nouns in the Czech press of the XVIIIth century
}

\section{[Les noms propres français dans la presse tchèque du XVIII ${ }^{\mathrm{e}}$ siècle]}

\author{
Zdenka Schejbalova
}

DOI: 10.18355/XL.2021.14.04.05

\begin{abstract}
Václav Matěj Kramerius (1753-1808) was a Czech publisher, writer, playwright and journalist, founder of modern Czech journalism.

His newspaper, named Krameriusovy c. k. vlastenecké noviny (Kramerius' ImperialRoyal Patriotic Newspaper), published since 1789, focused mainly on enlightenment of the common people, on national emancipation. Their main sections included official notices, announcements, foreign and domestic news, literature and theater news. One of the most important news were reports of revolutionary events in France at the end of the XVIIIth century (Great French Revolution).

The subject of this article is an analysis of the transcription method of French proper nouns in this Czech newspaper published by Kramerius. In order to simplify the reading of foreign names and place names, Kramerius tried to approximate their pronunciation as much as possible using the spelling system of the Czech language of his time. He used different ways such as phonetic transcription using Czech letters, changing or omitting French diacritics, using Czech diacritics (e.g., vowel length), changing the order of letters, omitting letters. Some proper nouns remain indeclinable, others are declinated according to their classification into declination types. Kramer's transcription also informs us about the pronunciation of French at that time, e.g., soft 1 $/ \lambda /$ is still in use, /we/ is already pronounced as /wa/.

Kramerius' newspaper is not only an important historical testimony of its time, but also provides valuable insights into the development of the Czech language.

Key words: V.M. Kramerius, Czech language, French proper nouns / names, spelling, Czech press of the XVIIIth century
\end{abstract}

\section{Résumé}

Václav Matěj Kramerius (1753-1808) était un éditeur, écrivain, dramaturge et journaliste tchèque, fondateur du journalisme tchèque moderne.

Son journal Krameriusovy c. k. vlastenecké noviny (Le journal impérial royal patriotique de Kramerius), publié à partir de 1789, se concentrait principalement sur l'éducation du peuple, sur l'émancipation nationale. Ses sections principales comprenaient des avis officiels, des annonces, des nouvelles étrangères et nationales, de la littérature et des nouvelles relatives au théâtre. Parmi les faits marquants, on compte les événements révolutionnaires en France à la fin du XVIII ${ }^{\mathrm{e}}$ siècle (Grande Révolution française).

Le sujet de cet article est une analyse de la méthode de transcription des noms propres français dans ce journal tchèque publié par Kramerius. Afin de simplifier la lecture des noms propres et des noms de lieux étrangers, Kramerius a essayé de s'approcher autant que possible de leur prononciation en utilisant le système orthographique tchèque de son époque. Il a recouru à différentes méthodes telles que la transcription phonétique en utilisant des lettres tchèques, en changeant ou en omettant les signes diacritiques français, en utilisant les signes diacritiques tchèques (par exemple, sur les voyelles longues), en omettant des lettres, en changeant leur ordre. Certains noms propres restent indéclinables, d'autres sont déclinés selon le modèle où ils sont classés. 
La transcription de Kramerius nous renseigne également sur la prononciation du français à cette époque : par exemple, le 1 mouillé / $\lambda$ / est toujours utilisé ; /wع/ est déjà prononcé comme /wa/.

Le journal de Kramerius est non seulement un important témoignage historique de son époque, mais il fournit également des informations précieuses sur le développement de la langue tchèque.

Mots-clés : V.M. Kramerius, langue tchèque, noms propres français, orthographe, presse tchèque du XVIII ${ }^{\mathrm{e}}$ siècle

\section{Introduction}

A la fin du XVIIIe et au début du XIXe siècle, après 200 ans d'oppression rigide, où l'utilisation de la langue tchèque a été restreinte au peuple asservi à la campagne, commence une période de renouveau, fervent et passionné, de la culture nationale tchèque.

Cet évènement se manifeste d'une façon marquante dans le développement de la presse périodique tchèque, dont la personnalité éminente était Václav Matěj Kramerius (Klimeš, 1955 : 70).

En 1789, Václav Matěj Kramerius, ancien professeur de langue tchèque, fonde son hebdomadaire „Krameriufowy c. k. pražfké pofftowské nowiny“ (Journal postal pragois, impérial et royal, de Kramerius), dénommé „Krameriufowy c. $k$. wlaftenské nowiny" à partir de 1791 (Journal patriotique, impérial et royal, de Kramerius), qui a un caractère rationaliste et reflète les idées du mouvement de Renouveau national tchèque (České národní obrození). Il représente un des premiers personnages essentiels de la presse tchèque, autant pour sa langue, son travail de rédaction ou ses manières journalistiques qui sont devenues des modèles pour les générations suivantes. Il introduit, par exemple, des informations sur la publication des livres, des rapports sur les lots de loterie, le prix des aliments et la rubrique „Hlasy čtenářu (Voix des lecteurs) -jamais auparavant, les lecteurs n'avaient eu la possibilité de s'exprimer dans un périodique. Les annexes à caractère instructif constituaient une autre innovation. Après la mort de Kramerius, en 1808, la maison d'édition continua encore d'exister pendant 17 ans, mais elle perdit en importance.

Grâce à son poste chez le chevalier Jan František Neuburk, amateur d'histoire et collectionneur passionné de vieux manuscrits tchèques, il a acquis une bonne connaissance de la langue tchèque. Il est devenu son bibliothécaire, copiste et imprimeur d'ouvrages précieux : c'est là qu'il a appris le métier d'imprimeur. Mais, par l'ampleur de ses lectures, il a dépassé le stade des notions pour développer une connaissance active du tchèque littéraire de la période d'avant 1620. Dans les livres qu'il lisait et qu'il réimprimait pour son patron, il a appris un tchèque correct et comment l'utiliser dans des œuvres, en maniant clarté et expressivité dans une langue littéraire (Rybička, 1859 : 9, Klimeš, 1955 : 71).

Sa culture en expression écrite n'avait pas d'égal. Le résultat de son effort fut la rédaction de plus de 30 volumes de notes grammaticales et de relevés phraséologiques, employés plus tard en tant que matériel lexical précieux par Josef Jungmann, quand ce dernier élaborait son dictionnaire tchèque-allemand (Novotný, $1973: 20)$.

Dans ses œuvres, il voulait s'approcher au plus près des lecteurs en employant une langue compréhensible, claire, simple et cordiale. Il s'est employé à utiliser une

XLinguae, Volume 14 Issue 4, October 2021, ISSN 1337-8384, eISSN 2453-711X 
langue tchèque „purifiée des balayures“ (od smetí vypulérovaném) (Beránková, 1981 : 41). La locution utilisée "psáti a mluviti po Krameriovsku" (écrire et parler à la Kramerius) signifiait bien écrire et bien parler tchèque (Rybička, $1859: 31$ ).

Son but est de relever la conscience humaine et nationale des fermiers et de petits artisans en leur prodiguant des conseils pratiques pour la vie quotidienne. Ce sont l'éducation et la formation du peuple qu'il met au premier rang et dans lesquelles il voit le moyen de base pour améliorer sa situation économique et culturelle (Beránková, 1981 : 39). Il parle à ses contemporains la langue de ses contemporains, une langue claire, simple, vivante et sans tendances archaïsantes. Tout en employant une langue populaire, Kramerius écrit un tchèque raffiné, ciselé pour son temps (Winter, $1936: 114-120$ ).

Kramerius encourage au patriotisme, à l'émancipation et à l'utilisation de la langue tchèque. Il énumère les nations slaves désignées comme des frères qui utilisent des langues peu différentes de la langue tchèque.

2.1.1790, n. 1, p.2

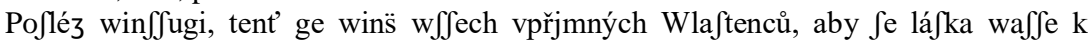

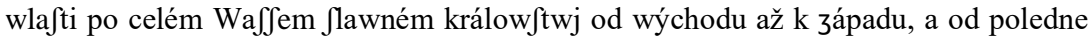

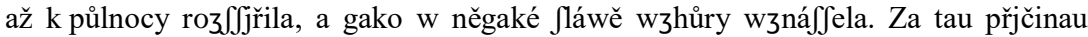

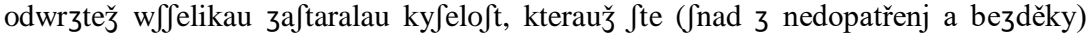

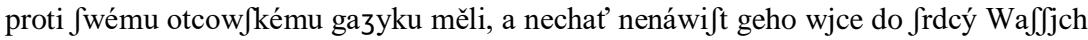

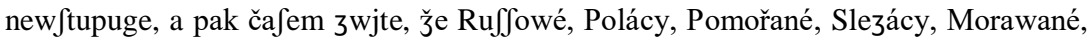
Slowácy, Dálmatowé, Bofñácy, Moldawané, Srbowé, Wendowé, Charwátowé, a

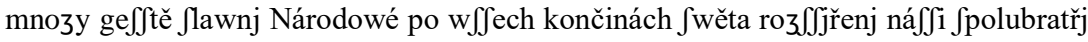

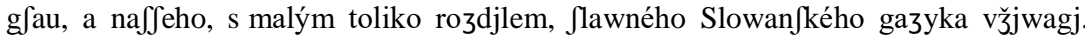
Spifowatel.

„Je désire ensuite, et c'est un désir de tous les patriotes sincères, que votre amour de la patrie se propage dans votre glorieux royaume tout entier, de l'est à l'ouest, et de midi à minuit, et qu'il monte haut, comme en gloire. Pour cette raison, rejetez toute sorte d'aigreur surannée que vous aviez (peut-être par méprise et involontairement) contre votre langue paternelle (sic); et que la rancour à son encontre n'entre plus dans vos cours; et puis, avec le temps, vous apprendrez que Russes, Polonais, Poméraniens, Silésiens, Moraves, Slovaques, Dalmates, Bosniaques, Moldaves, Serbes, Polabes (ici Wendes), Croates, et d'autres glorieuses nations encore répandues dans toutes les régions du monde sont nos frères et qu'ils se servent de notre glorieuse langue slave, avec de petites différences seulement. Écrivain. “"

Selon Kramerius, la compréhension de la langue slave (nommée ici slovenský) traverse les pays slaves, ce qui est, à son avis, unique au monde.

23.1.1790, č. 4

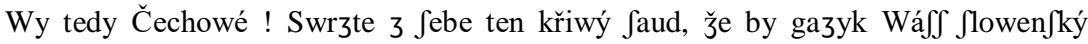
v malých toliko mezjch zawřen byl, anobř̌ chlubte $\int \mathrm{e}$ tjm a honofte flawně, že

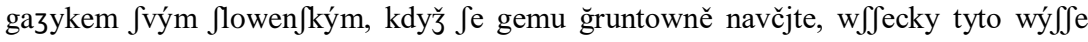
připomenuté země bezpečně progjti můžete, cő̌ se w prawdě žádnému 3wláft

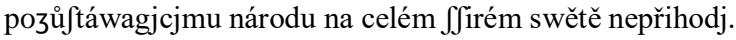


que votre langue slave soit enfermée dans d'étroites limites ; au contraire, glorifiezvous d'elle et faites-vous honneur de pouvoir passer en toute sécurité tous les pays rappelés plus haut avec votre langue slave si vous l'apprenez bien, ce qui n'arrive à aucune nation contemporaine du monde entier. "

Kramerius a également retenu le témoignage d'un Slovaque sur la reprise et la propagation de la langue slovaque d'une manière inconnue dans son histoire. Kramerius ajoute que, de même, le tchèque, presque disparu, se répand de façon à atteindre bientôt, selon lui, une place éminente.

Krameriusovy c.k. vlastenecké noviny, 27.3.1790, n. 13:

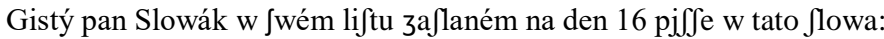

„Gakož nynj w Uher kém národu negednau láfka k Jtarému krogi obžiwla, tak také y

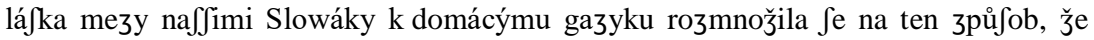

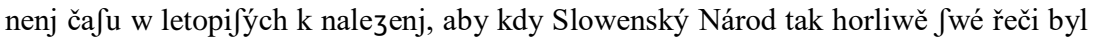
fe vgjmal gako nynj. Nepochybně gdau do Jebe, a vznáwagj, క̌e gim do cyzýho gazyka, gehož otcowé gegich neznali, nic nenj, a že mateřfká řeč předce neymileg $\int j \mathrm{j}$

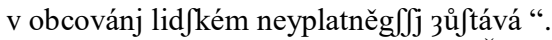

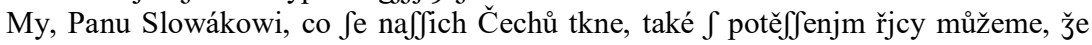

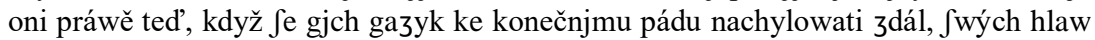

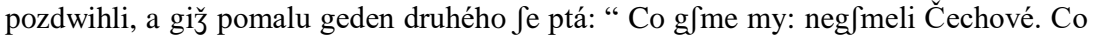

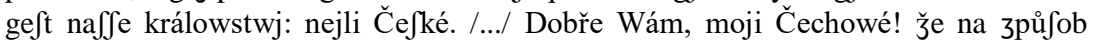
giných národů $\int$ wůg gazyk milugete, nebo gaké Wám dobré 3 gazyka toho, gemuక̌

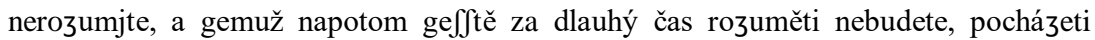
může.

„Un certain Slovaque écrit les mots suivants dans sa lettre du 16 :

Ainsi que l'amour du costume ancien a ressuscité à maintes reprises chez la nation hongroise, de même, parmi nos Slovaques, l'amour de la langue du pays s'est multiplié d'une manière telle qu'on ne peut trouver, dans les annales, d'autre époque où les Slovaques aient employé leur langue avec autant d'ardeur que maintenant. Assurément, ils ,rentrent en eux" et comprennent que la langue étrangère, que leurs pères ne connaissaient pas, n'est rien pour eux; et que leur très chère langue maternelle reste toutefois la plus utile dans la communication humaine.

Nous, à Monsieur le Slovaque, en ce qui concerne nos Tchèques, nous pouvons dire avec plaisir qu'ils ont relevé la tête au moment où leur langue semblait pencher vers sa fin ; et l'on se demande déjà l'un à l'autre: "Qui sommes-nous si nous ne sommes pas Tchèques? Qu'est-ce que notre royaume s'il n'est pas tchèque. /.../ C'est bien pour vous, mes Tchèques! qu'à la manière d'autres nations, vous aimiez votre langue, car quoi de bon peut vous provenir d'une langue que vous ne comprenez pas et que vous ne comprendrez toujours pas dans un futur lointain. "

Pour ses articles sur les événements à l'étranger, y compris sur la situation révolutionnaire en France, Kramerius puise dans la presse allemande (Beránková, 1981 : 42), mais aussi, par exemple, dans le Tableau des opérations de l'Assemblée nationale $^{l}$, dont il traduit les nouvelles, mot à mot.

La première nouvelle sur le bouleversement politique en France parait déjà le $1^{\mathrm{er}}$ août 1789 : Kramerius souligne que «Ve Frankrajchu ději se nyni takové věci, kteréž svou

\footnotetext{
${ }^{1}$ Tableau des opérations de l'Assemblée nationale : d'après le Journal de Paris : et auquel on a ajouté tous les articles de cette feuille, qui peuvent intéresser les arts \& les sciences, Lausanne : Chez Hignou \& Comp ${ }^{\mathrm{e}}$. Imprimeurs, 1789.
}

XLinguae, Volume 14 Issue 4, October 2021, ISSN 1337-8384, eISSN 2453-711X 
di̊ležitostí daleko i tureckou a švejdskou vojnu převyšují » (Novotný, 1973 : 100) "Il se passe en France des évènements dont l'importance dépasse de beaucoup même les guerres turque et suédoise. »

Le 15 août l'administration autrichienne de Vienne interdit de publier toute nouvelle sur la situation en France (Novotný, 1973 : 104). Cependant, l'édition du 18 août 1789 nous informe des évènements révolutionnaires du 16 juillet 1789, relatifs à la prise de la Bastille.

" ...lidu na tisice po ulicich jako vzteklých běhalo, a jeden druhého zabijel. Celé zástupy ozbrojeného lidu na bastillu (vězeni městské) dopadlo, mezi které tamši Ředitel z kuši a ručnic páliti dal ; »

« ... des milliers de gens couraient dans les rues, comme enragés, l'un tuait l'autre. Des foules entières de gens armés ont attaqué la Bastille (prison municipale), foules contre lesquelles le directeur des lieux a fait tirer à l'arbalète et au fusil ; ... »

À partir de 1792, les périodiques sont censurés et ensuite, après 1793, on diffuse une propagande antirévolutionnaire sur la terreur en France.

Le journal du 9 février 1793 rapporte la nouvelle de l'exécution du roi. Ce fait terrifiant a choqué toute l'Europe. Kramerius lui a consacré plus de la moitié de son périodique (six pages), où il a présenté en détail la fin tragique du roi.

„Dne 21 ho ledna tohoto 1793 roku... Ludvik XVI. toho jména, král francouzský, jehož někdy celý francouzský národ miloval skrze jeho dobré srdce, ctnosti a velikou lásku, kterouž měl k svému lidu, a jejž štastným učiniti žádal od roty prostopášného lidu ukrutně zamordován jest. Ten den ve všech francouzských zemích, kdež se již tolik mordi̊ a loupeži stalo, jest nejtruchlivějši a nejžalostnější. “

«Le 21 janvier de l'année 1793 ... le dénommé Louis XVI, roi de France, que jadis le peuple français aimait pour son bon cœur, ses vertus et le grand amour qu'il avait pour son peuple qu'il cherchait à rendre heureux, a été tué cruellement par une bande de gens licencieux. Ce jour, dans toutes les régions françaises où tant de meurtres et de vols se sont passés, est le plus triste et le plus affligeant. »

Kramerius, lui-même, réprouve les tueries d'innocents :

„,Tedy francouzšti tyranové ještě se vztekati neprestali: ještě tomu ukrutenství není konce, kteréž již toliknácte tisiců lidu zahubilo. - Hrưza jest psáti o těch všech nešlechetnostech, kterýchž se tento zaslepený lid den po dni více a více dopouští, a nemůž člověku jináč na mysl připadnout, než že Francouzové konečně sami sebe na záhubu přivésti chtějí. “

„Donc, les tyrans français n'ont pas encore cessé d'enrager: les cruautés n'ont pas encore pris fin, qui ont mis à mort des milliers de gens. Au vu de toutes ces immoralités que ce peuple aveuglé commet de plus en plus de jour en jour, il est horrible d'écrire qu'il ne peut que nous venir à l'idée que les Français veulent finir par s'anéantir eux-mêmes. »

C'est sa façon de transcrire les noms propres français dans les nouvelles sur la situation politique en France qui a retenu notre attention.

L'orthographe tchèque du XVIII ${ }^{\mathrm{e}}$ siècle suit la manière d'écrire issue de l'orthographe utilisée par l'Union des Frères Tchèques/Moraves (Unitas Fratrum) après l'invention de l'imprimerie, qui était, en grande partie, diacritique, tout en conservant quelques digrammes. Le point au-dessus des consonnes molles a été changé en caron (ou háček) $\check{c}, d$ ', $t$ ', $\check{n}, \check{z}, \check{r}$. La lettre $\check{s} / \int /$ n'était écrite comme $\ddot{s}$ qu'à la fin du mot (win $\ddot{s}=$ vinš) ; à l'initiale ou au milieu, on utilisait le digramme $\iint($ winffugi= vinšuji). Le 
graphème $\int$ rendait le phonème $/ s /$ à l'initiale et au milieu du mot $(g / a u=j$ jou $)$; à la fin, on écrivait $s$. La voyelle longue $i$ /i:/ était transcrite par $j$ (v̧̌jwagj=užívají). La consonne $/ j /$ avait le graphème $g$ ou $y$ (winffugi); pour la consonne $/ g /$, on se servait

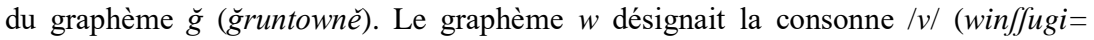
vinšuji), tandis que le graphème $v$ rendait la voyelle $/ u /$ à l'initiale du mot ( $v$ ǰjwagj $=$ užívají). La diphtongue $/ o u /$ était transcrite $a u(g / a u=$ jsou). Après $c, s, z$, on écrivait $y$ pour rendre la voyelle /i/ (Slowácy). (Gebauer, 1871 : 196-221)

\section{Analyse des noms propres}

Pour notre analyse des noms propres d'origine française utilisés dans les publications de Václav Matěj Kramerius, nous utilisons le classement des noms propres selon les critères de Rudolf Šrámek, qui les a formulés dans sa publication Úvod do obecné onomastiky. Rudolf Šrámek distingue trois plans principaux selon le type d'objet onomastique : géonymique, bionymique et chrématonymique; ils correspondent à la trichotomie de base des classes de noms propres : géonymes, bionymes, chrématonymes (Šrámek, 1999 :16).

\section{Bionymes}

Les bionymes désignent des objets vivants (ou prétendument vivants) tels que fixés par les relations sociales. Ils comprennent des anthroponymes, des "faux " anthroponymes, des théonymes, des zoonymes et des fytonymes.

Anthroponymes

Les prénoms, classés parmi les anthroponymes, ont la forme des équivalents en langue tchèque avec les signes orthographiques tchèques de l'époque (Jiřj, Ludvjk, Maximilián, Marye, Antonye $\rightarrow$ aujourd' hui Jiři, Ludvik, Maxmilián, Marie, Antonie), la voyelle longue $i$ /i:/ est transcrite par le graphème $j$.

\begin{tabular}{|l|l|}
\hline Jiřj Buton 16/8/94 & Georges Button \\
\hline Ludvjk Kapet 9/2/93 & Louis Capet \\
\hline Maximilián Robespier 16/8/94 & Maximilien Robespierre \\
\hline Marye Antonye 9/11/93 & Marie Antoinette \\
\hline
\end{tabular}

\section{Surnoms}

Pour s'approcher des révolutionnaires, Louis-Philippe d'Orléans s'est fait nommer par un nom commun, Égalité. Kramerius met son surnom Egalité entre parenthèses et le traduit en tchèque par l'équivalent Rovnost.

\begin{tabular}{|l|l}
\hline $\begin{array}{l}\text { Mě } \iint t \text { 'an Rovnost (Egalité) } \\
13 / 10 / 92\end{array}$ & $\begin{array}{l}\text { nom révolutionnaire de Louis - } \\
\text { Philippe d'Orléans }\end{array}$ \\
\hline
\end{tabular}

Noms propres collectifs

Dérivée à l'origine du hagionyme saint Jacques, la dénomination les Jacobins désignent une collectivité politique. En tchèque, le prénom hagionyme équivalent est

XLinguae, Volume 14 Issue 4, October 2021, ISSN 1337-8384, eISSN 2453-711X 
Jakub; d'où le nom collectif Jakubinowé.

\begin{tabular}{|ll|l|}
\hline Pař́̌̌́fftí Jakubinowé $\quad 20 / 10 / 92$ & Jacobins \\
\hline
\end{tabular}

Issu de Chouan, surnom de guerre d'un des chefs des insurgés royalistes de l'Ouest (Jean Chouan - Cottereau ; forme régionale de chat-huant), l'expression Šuani transcrit, en tchèque, la prononciation française, avec la désinence $-i$ du nominatif masculin pluriel.

\begin{tabular}{|l|l|}
\hline SJuani $3 / 10 / 95$ & Chouans \\
\hline
\end{tabular}

Patronymes

Les patronymes, y compris le nom de la terre adjoint au patronyme, sont, dans la plupart des cas, déclinés et transcrits phonétiquement en utilisant les caractères tchèques.

La particule dite nobiliaire $d e$, dont l'équivalent est en tchèque $z$, cesse d'apparaître après l'abolition de la noblesse par décret de l'Assemblée nationale le 23 juin 1790. Donc, en 1789, elle est encore présente ( $s$ Weywodau $z$ Willeynier, Marquisovi $z$ Fayette) ; plus tard, le patronyme reste sans particule (pro Pana la Fayete, Pan Lafayet). Néanmoins, dans certains cas, Kramerius mentionne toujours le titre de noblesse comte, prince (synové hraběte Artoa, hrabě Artoa, Prync Konde).

\begin{tabular}{|c|c|c|}
\hline Prync Konde & $31 / 12 / 91$ & \multirow[t]{2}{*}{ prince de Condé } \\
\hline Prync Konde & $20 / 10 / 92$ & \\
\hline na Pana Kondorceta & $23 / 7 / 91$ & Condorcet \\
\hline \multicolumn{2}{|c|}{ misto nich obrazowé Woltera a Rusoa 23/7/91 } & Voltaire, Rousseau \\
\hline synové hraběte Artoa & $29 / 9 / 92$ & Artois \\
\hline hrabě Artoa & $3 / 10 / 95$ & Artois \\
\hline za Drueta & $21 / 11 / 95$ & Drouet \\
\hline Generál Hofse & $20 / 8 / 96$ & Hoche \\
\hline Generál Bonapart Korfykán & $20 / 8 / 96$ & Bonaparte \\
\hline Generál Baron Bulie & $26 / 1 / 93$ & Louis de Bouillé \\
\hline
\end{tabular}

La transcription du patronyme Bouillé comme Bulie témoigne de la prononciation de $l$ mouillé $/ \hat{K}$ encore vivante en français, soit avant le passage à yod $/ \mathrm{j} /$.

Dans le cas du patronyme Jourdan, il y a la voyelle $a$, brève ou longue. La voyelle longue correspond mieux à la prononciation tchèque. Le phonème $/ \check{z} /$ est transcrit par le graphème tchèque du caron (ou háček) ž, tandis que le phonème vocalique /u/, écrit en français avec le digramme $o u$, est rendu par le graphème simple $u$.

\begin{tabular}{|l|l|}
\hline Generál Žurdan & \multirow{2}{*}{ général Jourdan } \\
\cline { 1 - 1 } Generál Žurdán & \\
\hline
\end{tabular}

La voyelle antérieure labialisée /y/ est transcrite soit par le graphème $y$, soit par $i$. 


\begin{tabular}{|ll|l|}
\hline Dymas & $16 / 8 / 94$ & Dumas \\
\cline { 1 - 2 } Sankt Žist & Saint Juste \\
\cline { 1 - 2 } St. Žist & $16 / 8 / 94$ & \\
\hline
\end{tabular}

La voyelle finale muette est généralement omise, sauf quelques exemples.

\begin{tabular}{|c|c|c|}
\hline Sankt Žist & $16 / 8 / 94$ & Saint Juste \\
\hline St. Žist & $16 / 8 / 94$ & \\
\hline \multicolumn{2}{|c|}{ Generál Bonapart Korfykán 20/8/96 } & Bonaparte \\
\hline Maximilián Robespier & $16 / 8 / 94$ & Maximilien Robespierre \\
\hline Generál Hofse & $20 / 8 / 96$ & Hoche \\
\hline Monteskye & $13 / 10 / 92$ & Montesquieu \\
\hline
\end{tabular}

L'exemple du patronyme composé avec l'adjectif saint sous la forme allemande de Sankt nous informe sur les sources des informations, qui étaient, en partie, la presse de langue allemande de Vienne ou de Francfort. Mais l'origine des nouvelles n'est indiquée que rarement. Cela n'était pas dans l'usage ou les habitudes.

Dans certains patronymes, il manque des lettres et tout le nom propre peut être altéré.

\begin{tabular}{|lc|l|}
\hline Hrabě Lally-Tolendl & $18 / 8 / 89$ & comte Gérard de Lally-Tollendal \\
\cline { 1 - 2 } Generál Pan Dumurier & $29 / 9 / 92$ & \multirow{2}{*}{ Charles-François du Périer Dumouriez } \\
\cline { 1 - 2 } Dumurierowa & & \\
\hline s Weywodau z Willeynier & $18 / 8 / 89$ & duc de Villequier \\
\hline Talirand & $30 / 11 / 99$ & Talleyrand \\
\hline
\end{tabular}

L'orthographe du patronyme du marquis de La Fayette est à chaque fois différente : on notera l'absence de l'article défini, celle de la préposition de, l'omission de lettres.

\begin{tabular}{|ll|l|}
\hline Marquisovi z Fayette & $18 / 8 / 89$ & \multirow{2}{*}{ marquis de La Fayette } \\
\cline { 1 - 2 } pro Pana la Fayete & $23 / 7 / 91$ & \\
\cline { 1 - 2 } Pan Lafayet & $29 / 9 / 92$ & \\
\hline
\end{tabular}

La transcription tchèque «oa » dans le nom du comte d'Artois témoigne déjà du changement de la prononciation /we/ en /wa/ qui se produit en français juste pendant cette période de la fin du XVIII ${ }^{\mathrm{e}}$ siècle.

\begin{tabular}{|ll|l|}
\hline synové hraběte Artoa & $29 / 9 / 92$ & Comte d'Artois \\
\cline { 1 - 2 } hrabě Artoa & $3 / 10 / 95$ & \\
\hline
\end{tabular}




\section{Géonymes}

Les géonymes sont tous les noms propres désignant des objets situés dans le paysage et fixés sur les cartes géographiques. Ils comprennent deux sous-catégories : toponymes et cosmonymes.

Les toponymes se divisent en oïkonymes (lieux habités), avec les sous-catégories urbonymes - ville, choronyme - région, pays, etc.; et en anoïkonymes (lieux nonhabités) avec les sous-catégories hydronymes - fleuves, oronymes - montagnes, etc.

Ö̈konymes - urbonymes (noms de villes)

Dans la plupart des urbonymes, nous trouvons la transcription phonétique modifiée selon l'orthographe tchèque (Saint-Cloud $\rightarrow$ do Klůdu - avec la voyelle longue $\stackrel{u}{ }$, typique du système vocalique tchèque, marquée d'un petit rond); les signes diacritiques français sont omis (SJalon - Châlon, w Makonu - Mâcon, SJambery Chambéry). Dans l'urbonyme Verdun, la voyelle nasale finale est transformée en voyelle longue $y$ et consonne nasale $n$ prononcées séparément. En tant que substantifs, ils sont déclinés, d'où différentes désinences vocaliques.

\begin{tabular}{|c|c|c|}
\hline Rems & $16 / 10 / 92$ & \multirow[t]{3}{*}{ Reims } \\
\hline z Remsu & $16 / 10 / 92$ & \\
\hline w Raymfu & $9 / 2 / 93$ & \\
\hline $\mathrm{Z} \mathrm{Mecu}$ & $29 / 9 / 92$ & \multirow[t]{2}{*}{ Metz } \\
\hline a mezy Mecem hradem Grymontem & $16 / 10 / 92$ & \\
\hline SJalon nad Marnau & $16 / 10 / 92$ & Châlon sur Marne \\
\hline SJambery & $20 / 10 / 92$ & Chambéry \\
\hline od Buloně & $3 / 10 / 95$ & Boulogne \\
\hline w Makonu & $10 / 12 / 91$ & Mâcon \\
\hline do Klůdu jeti & $30 / 7 / 91$ & \multirow[t]{2}{*}{ Saint-Cloud } \\
\hline do $\int w . ~ K l u d u$ & $30 / 11 / 99$ & \\
\hline pewnost Werdýn & $29 / 9 / 92$ & \multirow[t]{3}{*}{ Verdun } \\
\hline k Werdýnu & $20 / 10 / 92$ & \\
\hline pewnosti Werdýnu & $31 / 12 / 91$ & \\
\hline
\end{tabular}

Certains urbonymes marquent des modifications orthographiques importantes reflétant leur prononciation transformée. 


\begin{tabular}{|lc|l|}
\hline až k Dynkyrchu & $3 / 10 / 95$ & Dunkerque \\
\hline v Kompině & $9 / 8 / 94$ & Compiègne \\
\hline z Sejonu & $22 / 9 / 92$ & Soisson \\
\hline z Lifere & $16 / 8 / 94$ & Lisière \\
\hline na hradě tak řečeném de la Prusyer & $30 / 7 / 91$ & château de la Proustière \\
\hline k tak řečeném místu Thullieri & $30 / 7 / 91$ & Tuillerie \\
\hline z Bryslu & $17 / 12 / 91$ & Bruxelles \\
\hline
\end{tabular}

Anoïkonymes - hydronymes

Les noms de fleuves et de rivières connaissent des modifications orthographiques qui rendent la prononciation française (oi [wa] $\rightarrow$ Loaru) ou renvoient à la prononciation allemande déformée (Meynu, Sayny). Il y a aussi des écarts morphologiques. Les hydronymes du genre féminin La Loire, La Maine sont déclinés en tant que masculins, sauf La Marne qui reste au genre féminin (ici au cas instrumental). Dans les urbonymes composés d'un nom de fleuve ou de rivière, ce dernier est lié par la préposition nad (au-dessus de, c.-à-d. sur) qui exige le cas instrumental : Sfalon nad Marnau (Châlon sur Marne).

\begin{tabular}{|lc|l|}
\hline od dolní řeky Loaru & $3 / 10 / 95$ & La Loire \\
\hline řeku Más & $30 / 7 / 9$ & La Meuse \\
\hline od řeky Meynu & $29 / 9 / 92$ & La Maine \\
\hline a těla mrtwá do řeky Sayny wházeli & $29 / 9 / 92$ & La Seine \\
\hline SJalon nad Marnau & $16 / 10 / 92$ & Châlon sur Marne \\
\hline
\end{tabular}

Anoïkonymes - oronymes

La dénomination des Pyrénées se fait à l'aide du substantif féminin pluriel hory (montagnes) et de l'adjectif Pyrenejský (pyrénéen) accordé au féminin pluriel. L'adjectif Pyrenejský se forme sur le substantif féminin pluriel Pyreneje avec le suffixe - ský (à la forme masculine). Le groupe est, ici, décliné au génitif avec la préposition $z$ (de) et au locatif avec la préposition $w$ (dans). Dans la racine, on observe l'alternance y/i (PyreneyJkých/ Pireneyfkých).

L'oronyme Les Ardennes est au datif masculin pluriel Ardenům, tandis que le tchèque contemporain le considère comme de genre féminin (soit, au datif féminin pluriel, Ardenám).

\begin{tabular}{|lc|l|}
\hline z Pireneyfkých hor & 23/7/91 & \multirow{2}{*}{ Les Pyrénées } \\
\cline { 1 - 2 } w Pyreneyfkých horách & $3 / 10 / 95$ & Les Ardennes \\
\hline díl Ardenům & $26 / 1 / 93$ & \\
\hline
\end{tabular}

Choronymes : noms de pays, noms de régions

Le nom de pays La France apparait tout d'abord sous la forme masculine venue de la version allemande (Frankreich $\rightarrow$ ve Frankrajchu) avec la prononciation transcrite en tchèque ; ensuite, nous trouvons la forme masculine au pluriel Francouzy, tandis qu'aujourd'hui le choronyme Francie est au singulier du genre féminin. 
Le nom de la région La Champagne reçoit à l'initiale le son consonantique /3/

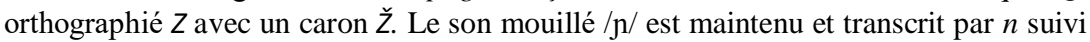
par $y$. L'orthographe contemporaine tchèque le transcrit avec $/ \mathrm{J} /$ à l'initiale et $/ \mathrm{n} /$ à la fin : Šampan̆.

La région de genre féminin La Savoie reçoit le suffixe - sko ; elle passe ainsi au genre neutre (ici, au cas génitif). Il est intéressant de voir la lettre $-\mathrm{f}$ - à la place de $-\mathrm{v}-$, aujourd'hui : Savojsko.

Pour ce qui est de la région de La Vendée, la transcription tchèque présente l'omission de l'accent aigu et de e muet final.

\begin{tabular}{|lc|l|}
\hline Ve Frankrajchu & $1 / 8 / 89$ & La France \\
\hline zpráwy z Francauz & $19 / 11 / 91$ & La France \\
\hline & & \\
\hline w Žampanyi & $20 / 10 / 92$ & La Champagne \\
\hline z Safoyfka & $20 / 10 / 92$ & La Savoie \\
\hline w kragině Wende & $10 / 12 / 91$ & La Vendée \\
\hline
\end{tabular}

Nésonymes : noms propres d'îles

Le nom de l'île de Noirmoutier est transcrit phonétiquement, ou - /u/, ier - /je/ et déjà avec la prononciation de $o i-/ \mathrm{wa} /$. Dans le cas de la dénomination des îles Guadeloupe et Saint-Dominique, nous pouvons remarquer une altération, où manquent des lettres : Gadalupe, Domnyk. L'abréviation usuelle $S w$. renvoie à l'adjectif tchèque swatý (saint).

\begin{tabular}{|lc|l|}
\hline kragině Noarmutje & 21/11/95 & l'île de Noirmoutier \\
\hline z oftrowa Sw. Lucye a Gadalupe & $24 / 12 / 91$ & Saint-Lucie et Guadeloupe \\
\hline na oftrowě Sw. Domnyka & $24 / 12 / 91$ & Saint-Dominique \\
\hline
\end{tabular}

Pour certains choronymes, il y a déjà des formes adaptées et tchéquisées : Pařiž (Paris), Strasburk (Strasbourg), Lucemburk (Luxembourg), Francouzy (la France), Bretan̆ (la Bretagne).

\section{Conclusion}

Václav Matěj Kramerius, en informant en détail sur la situation politique en France, s'efforce de rapprocher le plus possible les anthroponymes et les toponymes du système orthographique de la langue tchèque de son époque et, ainsi, de faciliter la lecture des noms étrangers auprès de ses lecteurs tchèques.

Les modifications orthographiques que nous avons relevées sont les suivantes : transcription phonétique à l'aide des lettres tchèques, - changement ou omission des signes diacritiques français, - introduction des signes diacritiques tchèques (sur les voyelles longues), - changement de l'ordre des lettres, - omission de lettres. Toutes les consonnes finales transcrites sont prononcées. La transcription nous instruit également sur la prononciation française de l'époque (l mouillé / $K /$ encore maintenu ; /we/ passe déjà à /wa/).

Pour des questions de déclinaison, la forme orthographique de la lettre finale peut changer selon le type flexionnel (masculin, féminin, neutre) ou disparaître si elle ne se prononce pas. 
Le genre, souvent masculin, diffère de l'usage moderne. Soit le nom propre reste indéclinable, soit il est décliné au genre féminin, masculin ou neutre.

Václav Matěj Kramerius est considéré comme un éminent journaliste, éditeur éclairant et patriotique, éveilleur du peuple dans les Pays tchèques du XVIII ${ }^{\mathrm{e}}$ siècle. Grâce à son journal tchèque et à ses publications, des idées de progrès social et d'instruction générale ont commencé à se répandre parmi le peuple tchèque. Dans toutes ses activités, il a visé l'éducation politique et culturelle de la classe moyenne et des paysans tchèques pour encourager leur fierté civique et nationale, leur ferveur patriotique. De plus, il a contribué considérablement à fixer la langue littéraire tchèque, instable encore à l'époque.

\section{Bibliographic references}

BERANKOVA, M. 1981. Dějiny československé žurnalistiky. D. 1, Český periodický tisk do roku 1918 : Československé dějiny žurnalistiky (Variant.). Praha: Novinář.

GEBAUER, J. 1871. Př́spěvky k historii českého pravopisu a výslovnosti staročeské. Praha.

KLIMES, V. 1955. Počátky českého a slovenského novinářství. 1. vyd. Praha: Orbis. NOVOTNY, J. 1973. Matěj Václav Kramerius. Praha : Melantrich.

RYBICKA, A. 1859. Život a působení Václava Matěje Krameriusa. Praha: J. Pospíšil. SOLLE, Z. 1998. Století české politiky :počátky moderní české politiky od Palackého a Havlíčka až po realisty Kaizla, Kramáře a Masaryka. Praha: Mladá fronta. ISBN 80204-0696-4.

SRAMEK, R. 1999. Úvod do obecné onomastiky. Brno : MU.

Tableau des opérations de l'Assemblée nationale : d'après le journal de Paris : et auquel on a ajouté tous les articles de cette feuille, qui peuvent intéresser les arts \&les sciences, Lausanne: Chez Hignou \& Comp ${ }^{\mathrm{e}}$. Imprimeurs, 1789. Répéré à http://books.google.fr/books?id=IRBAAAAAcAAJ\&pg=PA187\&lpg=PA187\&dq=du $\mathrm{c}+\mathrm{de}+$ Villeroy, + Bailly\&source=bl\&ots=W4Kq0IQgdK\&sig=AsESZQcJc90bwbw867 KLnD1_KWM\&hl=cs\&sa=X\&ei=iAcWVKDaGqzb7AaBmIHQCw\&ved=0CEkQ6A EwBQ\#v=onepage \&q=duc\%20de $\% 20$ Villeroy $\% 2 C \% 20$ Bailly $\& \mathrm{f}=$ false.

VOLF, J. 1930. Dějiny novin v Čechách do r. 1848. Praha: Nákladem vydavatelstva Ducha novin.

WINTER, G. 1936. Poznámky k novinářské češtině (Podněty k diskusi), Slovo a slovesnost, année 2, n. 2, pp. 114-120.

Les numéros du périodique Krameriusovy c.k. vlastenecké noviny analysés :

$1 / 8 / 89,18 / 8 / 89,2 / 1 / 90,27 / 3 / 90,23 / 7 / 91,30 / 7 / 91,19 / 11 / 91,10 / 12 / 91,17 / 12 / 91$, 24/12/91, 31/12/91, 22/9/92, 29/9/92, 13/10/92, 16/10/92, 20/10/92, 26/1/93, 9/2/93, 9/11/93, 9/8/94, 16/8/94, 3/10/95, 21/11/95, 20/8/96, 30/11/99.

Available online: http://kramerius.nkp.cz/kramerius

Words: 4878

Characters: 33934 (18,85 standard pages)

PhDr. Zdeňka Schejbalová

Faculty of Education

Masaryk University

Brno

Czech Republic

schejbalova@ped.muni.cz 